\title{
A supplementary benefit?
}

For the first time in the 23 years of publication it has proved necessary to produce a supplement for Pediatric Radiology. The supply of excellent papers, which has been rising steadily for several years, has recently exceeded the capacity of the regular issues. The status of all the papers published by Pediatric Radiology is equal - all have been peer reviewed and will be properly referenced in the world medical literature - so the only real difference between this supplement and the usual issue is in size.

As of 1996, starting with volume 26, the number of issues will be increased, but together with the publisher we came to the conclusion that a supplement would be the most effective method of dealing with the sizeable backlog. There will, of course, be constant monitoring to avoid a renewed build-up of papers awaiting publication. A number of other international journals have found themselves in this situation over the years. In our case it may be related to the unique status of this journal. Pediatric radiology has grown at an enormous rate across the world and has eagerly accepted, evaluated and developed the new imaging modalities. The centers of excellence have mushroomed, and from them have come increasing numbers of interesting and innovative papers. It is also not surprising that the need to publish has spread throughout the world. In this age of electronic publishing, the announcement of the death of the printed journal may have been somewhat premature!

The Editors 